\title{
Myocardial perfusion PET: A split function?
}

\author{
Riemer H. J. A. Slart, MD, PhD
}

\section{See related article, pp. 650-656}

Positron emission tomography (PET) plays an important role in the assessment of myocardial blood flow (MBF). Absolute quantification of MBF is feasible using tracer kinetic models. ${ }^{1}$ The most common method for quantifying $\mathrm{MBF}$ involves the acquisition of dynamic images with the cyclotron-produced radiopharmaceuticals ${ }^{13} \mathrm{~N}$-ammonia and ${ }^{15} \mathrm{O}$-water. An alternative method is the use of the generator produced ${ }^{82} \mathrm{Rb}$.

Several cardiovascular disorders have been evaluated with MBF PET. For instance, MBF measured by PET has been undertaken in patients with coronary artery disease (CAD), hypertrophic cardiomyopathy (HCM), idiopathic dilated cardiomyopathy (IDCM), and microvascular disease. ${ }^{2-5}$

Also, important long-term prognostic value of reduced coronary flow reserve in estimated with PET has been reported. ${ }^{5-7}$ In other words, MBF assessment provides a way to document and to estimate the risk of cardiovascular events. The majority of these previous studies are based on the MBF measurement of the entire myocardial wall.

In this issue of the Journal of Nuclear Cardiology, Rutten-Vermeltfoort and colleagues present interesting data regarding the determination of subendocardial and subepicardial MBF in 27 healthy subjects assessed with ${ }^{15}$ O-labeled water. ${ }^{8}$ An additional MRI was performed to measure left ventricular volumes and function. They found that mean resting $\mathrm{MBF}$ in the subendocardium was higher than the subepicardium level. Of interest,

From the Department of Nuclear Medicine and Molecular Imaging, Cardiovascular Imaging Group Groningen, University Medical Center Groningen, University of Groningen, Groningen, The Netherlands.

Reprint requests: Riemer H. J. A. Slart, MD, PhD, Department of Nuclear Medicine and Molecular Imaging, Cardiovascular Imaging Group Groningen, University Medical Center Groningen, University of Groningen, Hanzeplein 1, P.O. Box 30001, 9700 RB Groningen, The Netherlands; r.h.j.a.slart@ngmb.umcg.nl.

J Nucl Cardiol 2011;18:544-6.

$1071-3581 / \$ 34.00$

Copyright (C) 2011 The Author(s). This article is published with open access at Springerlink.com

doi:10.1007/s12350-011-9381-0 during adenosine stress, vasodilation was augmented to a greater extent in the subepicardium compared with the subendocardium. The subendocardial-to-subepicardial MBF ratio decreased significantly during hyperemia $(1.35 \pm 0.23$ to $1.12 \pm 0.20, P<0.001)$. Hyperemic transmural MBF was inversely correlated with left ventricular end-diastolic volume index $\left(r^{2}=0.41\right.$, $P=0.0003$ ), with a greater impact at the subendocardial level. The authors concluded that a transmural perfusion gradient can be detected in normal healthy subjects in a routine clinical setting with ${ }^{15} \mathrm{O}$-water PET.

Evaluation of MBF in several cardiovascular disorders as mentioned previously can be optimized in a different way by applying a split up in subendocardial and subepicardial regions. As mentioned by the authors the transmural perfusion gradient can be determined in patients with hypertrophic hearts or in patients with cardiac syndrome $\mathrm{X}$, for detecting microvascular dysfunction. ${ }^{4,9}$

For several reasons this study also offers opportunities for additional clinical applications, such as the unravelling of the etiology of transient ischemic dilatation (TID). An abnormal increase in TID ratio is regarded as an important marker that may indicate extensive CAD. It is described as an enlargement of the left ventricle (LV) cavity during stress due to extensive subendocardial ischemia that normalizes at rest. TID is frequently described as a combination of true physical dilatation of the end-diastolic volume, and apparent dilation caused by non-visualization of the sub-endocardium or by heart rate acceleration. ${ }^{10}$

Evaluation of subendocardial infarction is another field of interest. The method described in this study may assist in a better functional delineation of both layers of the myocardial wall. Up to now, MRI has played the leading role in the evaluation of subendothelial infarction. However, the anatomical and functional combination of PET-CT or even PET-MRI MBF with a contrast agent may replace the MRI approach. Absolute quantification of MBF is a strong tool of PET imaging, and the better delineation of the subendocardium by the current method in combination with $\mathrm{CT}$ may put PET-CT on a higher level.

Further, one would suspect that cardiac resynchronization therapy (CRT) may affect the transmural flow gradient. If so, relating the subendocardium-to-subepicardium ratio to LV function may be used to evaluate or even predict the LV response on CRT. 
Finally, the quantification of regional myocardial blood flow may be clinically useful not only for assessing the extent and severity of CAD, but also to detect and measure impairments in microcirculatory function in non-coronary cardiac disease. The prognostic significance of transmural microcirculatory dysfunction in the absence of CAD however remains to be assessed.

A number of technical aspects in this study need to be taken into account. As mentioned by the authors, tissue surrounding the myocardium could influence perfusion values and lead to heterogeneity of the perfusion pattern. Region definition of smaller areas of interest, such as with subendocardial and subepicardial flow measurements, will introduce more noise and potentially augment spillover artifacts from adjacent tissue. Moreover, spillover of activity between myocardial layers does occur. Simulation models and in vivo studies have revealed that the latter will result in underestimation of the transmural gradient.

The limited spatial resolution remains an important drawback for accurate measurements (i.e., without bias) of subendocardial and subepicardial MBF using PET. In this study a PET scanner with moderate spatial resolution was used. In the last 2 decades, however, the spatial resolution of PET scanners has significantly improved (from $>16$ to $<4 \mathrm{~mm}$ FWHM) and the impact of patient movement on quantification becomes more critical as the resolution increases. This is an area of active development and automated robust solutions for this long-standing issue should become available in the next years.

It is also clear that resolution is not the only limiting factor. Cardiac and respiratory movements pose a limitation on the effective resolution achievable. Gating (list) mode using ECG and respiratory signal was lacking in this study and therefore poses a serious limitation. This will need to be applied in the future for optimal delineation of the subendocardial and subepicardial layers.

Generator produced ${ }^{82} \mathrm{Rb}$ for MBF estimation is of more general use in imaging centers, especially in USA. The positron range, however, of ${ }^{82} \mathrm{Rb}$ is longer than ${ }^{13} \mathrm{~N}$-ammonia and ${ }^{15} \mathrm{O}$-water and will result in suboptimal image resolution. Therefore, the determination of subendocardial and subepicardial MBF with ${ }^{82} \mathrm{Rb}$ PET should be evaluated before it is applied clinically. Also, PET MBF protocols will differ between imaging centers, for instance the use of dynamic or static images. Static images have a higher (better) signalto-noise ratio, but the subendocardial-to-subepicardial MBF ratio may be reduced due to redistribution of the tracer. On the other hand, will MBF modelling need to be redefined in dynamic imaging? Will the delineation of different borderzones influence regional perfusion results and should we thus redefine normal perfusion and MPR values?

Previously, the determination of subendocardial and subepicardial MBF was validated mainly by animal experiments and microspheres. ${ }^{11}$ Of importance is the reproducibility of the transmural perfusion gradient, both test-re-test and between centers. This needs to be addressed in future studies.

Finally, why only MBF for measurement in the subendocardium versus the subepicardium? Of interest may be metabolic imaging. FDG-PET, or even (neuro) receptor imaging might benefit from assessing transmural gradients. Myocardial ischemia and viability may also to be linked with metabolic ${ }^{12}$ and neuro-receptor gradient changes.

In summary, the data of this study are encouraging. PET-(CT) MBF measurements may have additional clinical value using the transmural myocardial perfusion gradient. This "split function" method needs to be further optimized before optimal clinical utility.

\section{Open Access}

This article is distributed under the terms of the Creative Commons Attribution Noncommercial License which permits any noncommercial use, distribution, and reproduction in any medium, provided the original author(s) and source are credited.

\section{References}

1. Hutchins GD, Schwaiger M, Rosenspire KC, et al. Noninvasive quantification of regional blood flow in the human heart using N-13 ammonia and dynamic positron emission tomographic imaging. J Am Coll Cardiol 1990;15:1032-42.

2. Camici PG, Crea F. Coronary microvascular dysfunction. N Engl J Med 2007;356:830-40.

3. de Jong RM, Tio RA, van der Harst P, et al. Ischemic patterns assessed by positron emission tomography predict adverse outcome in patients with idiopathic dilated cardiomyopathy. J Nucl Cardiol 2009;16:769-74.

4. Knaapen P, Germans T, Camici PG, et al. Determinants of coronary microvascular dysfunction in symptomatic hypertrophic cardiomyopathy. Am J Physiol Heart Circ Physiol 2008;294: H986-93.

5. Tio RA, Dabeshlim A, Siebelink HM, et al. Comparison between the prognostic value of left ventricular function and myocardial perfusion reserve in patients with ischemic heart disease. J Nucl Med 2009;50:214-9.

6. Herzog BA, Husmann L, Valenta I, et al. Long-term prognostic value of $13 \mathrm{~N}$-ammonia myocardial perfusion positron emission tomography added value of coronary flow reserve. J Am Coll Cardiol 2009;54:150-6.

7. Slart RH, Zeebregts CJ, Hillege JL, De Sutter J, Dierckx RA, van Veldhuisen DJ, et al. Myocardial perfusion reserve after a PETdriven revascularization: a strong prognostic factor. J Nucl Med (in press). 
8. Vermeltfoort IA, Raijmakers PG, Lubberink M, Germans T, van Rossum AC, Lammertsma AA, et al. Feasibility of subendocardial and subepicardial myocardial perfusion measurements in healthy normals with ${ }^{15} \mathrm{O}$-labeled water and positron emission tomography. J Nucl Cardiol. doi:10.1007/s12350-011-9375-y.

9. de Vries J, DeJongste MJ, Jessurun GA, et al. Myocardial perfusion quantification in patients suspected of cardiac syndrome $\mathrm{X}$ with positive and negative exercise testing: A [13 N]ammonia positron emission tomography study. Nucl Med Commun 2006;27:791-4.

10. van der Veen BJ, Kuperij N, Stokkel MP. Transient ischemic dilatation ratio derived from myocardial perfusion scintigraphy: What are we looking at? J Nucl Cardiol 2010;17:207-15.
11. Rimoldi O, Schafers KP, Boellaard R, et al. Quantification of subendocardial and subepicardial blood flow using ${ }^{15} \mathrm{O}$-labeled water and PET: Experimental validation. J Nucl Med 2006;47:163-72.

12. Southworth R, Dearling JL, Medina RA, et al. Dissociation of glucose tracer uptake and glucose transporter distribution in the regionally ischaemic isolated rat heart: Application of a new autoradiographic technique. Eur $\mathrm{J}$ Nucl Med Mol Imaging 2002;29:1334-41. 